\title{
Follicular Occlusion Triad: A Case Report
}

\author{
Chung-Min Yoon ${ }^{1}$ (D), Jung-Ha Kwak ${ }^{1}$ (D), Song-Hee Han² (D), Ji-An Choi ${ }^{1}$ (D) \\ Departments of ${ }^{1}$ Plastic and Reconstructive Surgery and ${ }^{2}$ Pathology, Dong-A University School of Medicine, Busan, Korea
}

\begin{abstract}
Follicular occlusion triad (FOT) is a complex chronic inflammatory skin disease comprising hidradenitis suppurativa (HS), acne conglobata (AC), and dissecting cellulitis of the scalp (DCS; Hoffman's disease or perifolliculitis capitis abscedens et suffodiens). While pathological mechanisms are responsible for common skin manifestations, the exact underlying causes of follicular occlusion have not yet been clearly identified. Therefore, the diagnosis and treatment of FOT remain challenging. A 31-year-old man on conservative treatment for previously diagnosed HS and AC presented to our clinic with multiple masses on his posterior neck and face. Excisional biopsy of the masses revealed epidermal cysts. Four months after the surgery, he presented with a painful palpable mass around the occipital region of the scalp with characteristic skin manifestations such as cicatricial alopecia and comedones and was diagnosed with DCS. Incision and drainage of the lesion were performed, and histopathology revealed pathological findings of follicular occlusion. The patient was diagnosed with FOT. Following the procedure, the patient has been on regular follow-up and is on oral isotretinoin; there have been no complications for the last 6 months.
\end{abstract}

Keywords: Hidradenitis suppurativa; Acne conglobata; Perifolliculitis capitis abscedens et suffodiens

\section{Introduction}

Follicular occlusion triad (FOT) refers to a complex chronic inflammatory skin disease comprising hidradenitis suppurativa (HS), acne conglobata (AC), and dissecting cellulitis of the scalp (DCS; Hoffman's disease or perifolliculitis capitis abscedens et suffodiens) [1]. The pathophysiology of FOT disease involves hair follicle occlusion, which causes the characteristic symptoms [1]. These symptoms can significantly compromise the patient's quality of life. Hence, it important to minimize the aggravation and recurrence of symptoms through accurate diagnosis and appropriate treatment. However, the exact underlying causes of follicular occlusion remain obscure, and the diagnosis and treatment of FOT are still challenging [1].

Herein, we present a case report about our experience of diagnosing and treating DCS and FOT in a patient who was on treatment for HS and AC. We also review the literature related to the disease The study was approved by the Institutional Review Board of Dong-A University Hospital (IRB No. DAUHIRB-21-023) and performed in accordance with the principles of the Declaration of Helsinki. Written informed consent was obtained from the patient for publishing the clinical images.

\section{Case}

A 31-year-old male patient visited our outpatient clinic with symptoms of multiple masses on both upper eyelids and on the posterior neck that developed a year ago. At 13 years of age, he had been diagnosed with $\mathrm{AC}$ on the face and chest, and at 18 years, he had been diagnosed with bilateral axillary HS.

\section{Case Report}

Received: March 23, 2021

Revised: June 5, 2021

Accepted: June 6, 2021

Corresponding author: Ji-An Choi, M.D.

Department of Plastic and Reconstructive Surgery, Dong-A University School of Medicine, 26 Daesingongwon-ro, Seo-gu, Busan 49201, Korea

Tel: +82-51-240-5416

Fax: +82-51-243-5416

E-mail: eyeball84@naver.com

This is an Open Access article distributed under the terms of the Creative Commons Attribution Non-Commercia License (https://creativecommons.org/licenses/by-nc/4.0/) which permits unrestricted non-commercial use, distribution, and reproduction in any medium, provided the original work is properly cited.

C) 2021 Korean Wound Management Society 
The patient was obese with a body mass index of $32 \mathrm{~kg} / \mathrm{m}^{2}$ and smoked 20 cigarettes per day. Previous surgical history included bilateral hip replacement for avascular necrosis of the femoral heads 4 years ago. The patient had no persisting symptoms or complications following the surgery. At the time of presentation, his medications included cefroxadine $250 \mathrm{mg}$ per os (PO) every 8 hours and isotretinoin $10 \mathrm{mg}$ PO every 12 hours. On physical examination, characteristic skin lesions of AC such as numerous comedones, cystic nodules, pustules, and depressed or keloidal scars were seen on his face, chest, and back. In addition, open tombstone comedones and bridged scars characteristic of HS were observed in both axillae (Fig. 1).

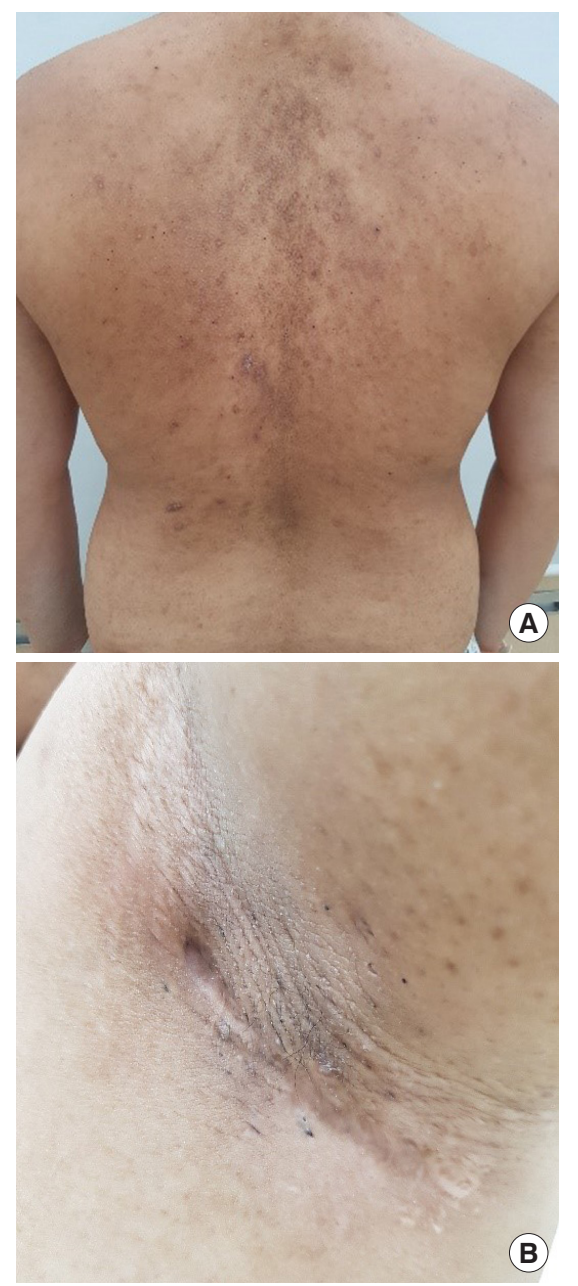

Fig. 1. Clinical photographs taken at initial presentation. (A) Characteristic skin lesions of acne conglobata such as numerous comedones, cystic nodules, pustules, and depressed or keloidal scars are observed on the patient's back. (B) On the left, axilla, open tombstone comedones and bridged scar lesions characteristic of hidradenitis suppurativa are observed.
Painless nodules approximately $1 \mathrm{~cm}$ in size were observed on both upper eyelids. Behind his neck, there was a diffuse, painful soft-tissue swelling with indistinct borders and a skin lesion $0.2 \mathrm{~cm}$ in size with slight oozing of blood (Fig. 2). Preoperative ultrasound imaging showed multiple epidermal cysts on both upper eyelids and a ruptured epidermal cyst in the posterior neck. Excisional biopsy was performed on the eyelid lesions under local anesthesia. Operative findings showed several cystic masses with distinct borders covered by an epidermal layer, including one on the right upper eyelid and two on the left upper eyelid. Following the eyelid surgery, an incision was made over the posterior neck mass through the skin opening, and exploration revealed a $4-\mathrm{cm}$-long sinus, containing pus and necrotic tissue but without clear demarcation from the surrounding fibrous tissue.

Necrotic tissue around and within the sinus was surgically debrided. The excised tissue was sent for histopathological examination, and a pus sample was sent for Gram staining and bacterial culture. Extensive irrigation was performed using betadine and normal saline, and direct closure was performed. Histopathological examination confirmed epidermal cysts in both nodules of the upper eyelid and ruptured epidermal cysts with foreign body reaction and abscesses in the posterior neck mass. The pus culture showed growth of Streptococcus agalactiae, and the patient was administered cefaclor $250 \mathrm{mg}$ PO every 8 hours for 2 weeks. Stitches were removed after 1 week, and the wound had healed without any acute complications.

The patient revisited our clinic 4 months after the surgery, complaining of painful palpable masses with yellowish discharge in the occipital region of the scalp. An $8 \times 5 \mathrm{~cm}$-sized mass was palpable at the site, which was about $4 \mathrm{~cm}$ above the

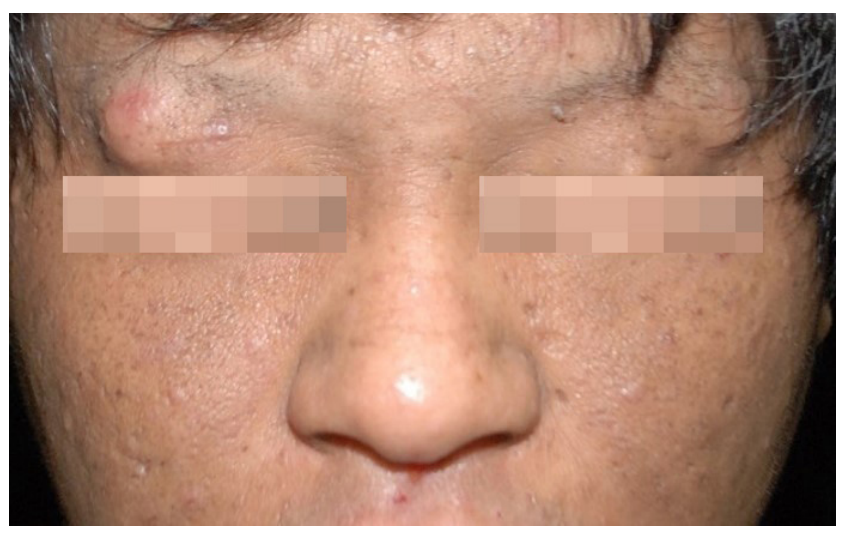

Fig. 2. Clinical photograph taken at initial presentation. Multiple nodules along with characteristic skin lesions of acne conglobata are observed on the patient's face. 


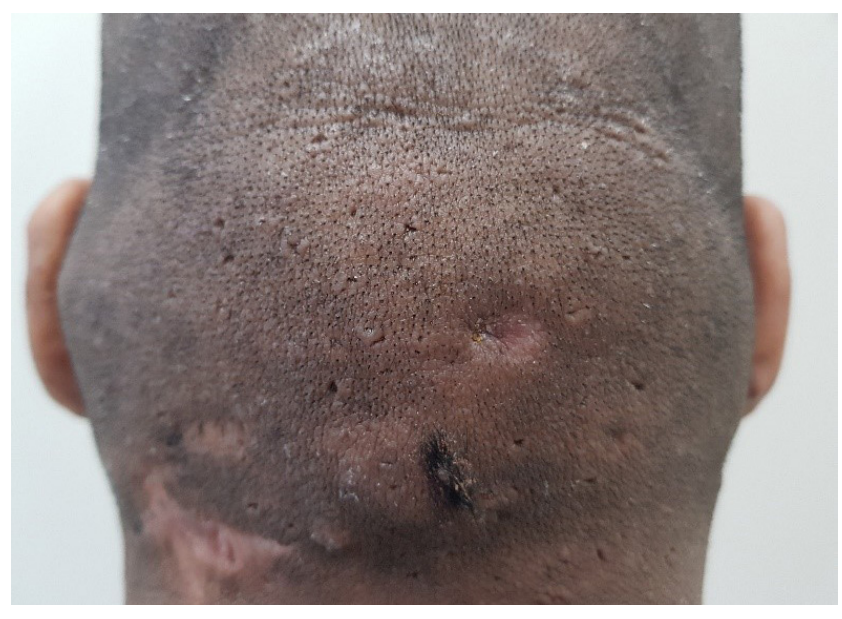

Fig. 3. Preoperative findings. Characteristic skin lesions of dissecting cellulitis of the scalp including cicatricial alopecia, polytrichia, comedones, and plugging with dried yellow crust are observed on the occipital region of the scalp.

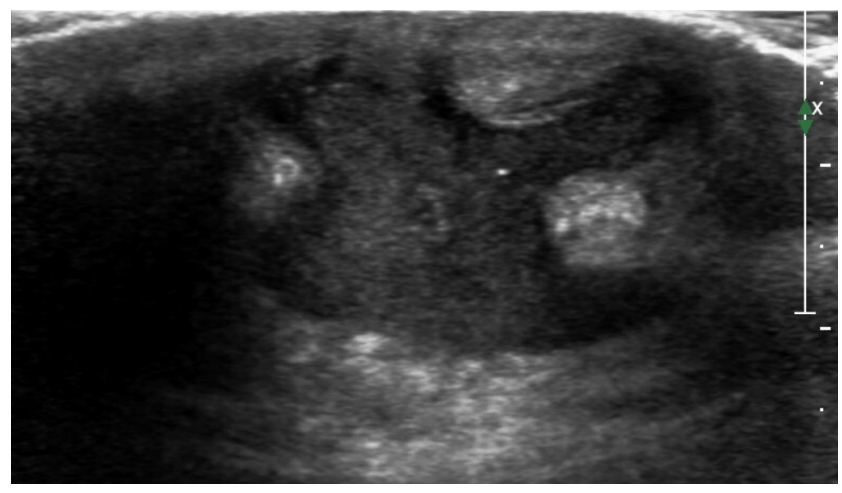

Fig. 4. Preoperative color Doppler ultrasound imaging. Ultrasound imaging shows irregularly shaped multiple masses containing floating materials.

previous posterior neck lesion. The patient's hair on the scalp was shaved for better visualization of the masses, and cicatricial alopecia, polytrichia, comedones, and plugging with dried yellow crusts were observed (Fig. 3). Color Doppler ultrasound imaging demonstrated increased vascularity within the subcutaneous fat area as well as multiple irregular-shaped masses with floating materials inside, which indicated multiple infected ruptured epidermal cysts (Fig. 4).

We performed a 5-cm incision over the center of the palpable occipital mass. Operative findings revealed about eight sinuses, each measuring $1 \mathrm{~cm}$ in length with extensive surrounding scar tissue without clear demarcation. The sinus discharge contained gel-like necrotic debris mixed with pus, and each sinus was interconnected to the other, forming tracts within the lesion (Fig. 5). Debridement and extensive irriga-

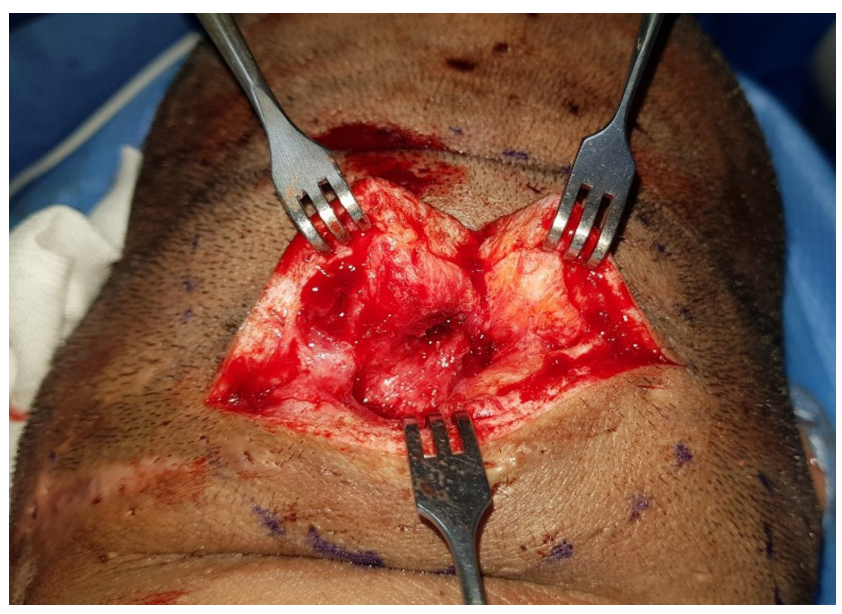

Fig. 5. Intraoperative findings. Operative findings show draining sinus tracts in a honeycomb pattern, which are characteristic of follicular occlusion triad.

tion with doxycycline, betadine, and normal saline were performed. Pathological examination and bacterial cultures of the retrieved tissue and discharge samples were performed, respectively. The wound was left open for a week after the surgery, and the dressing was changed twice daily. The bacterial cultures were positive for Staphylococcus epidermidis and Staphylococcus aureus. Following consultation with an infectious disease specialist, $1 \mathrm{~g}$ cefazolin was administered intravenously every 8 hours.

One week later, delayed wound closure was performed after confirming negative wound cultures. No complications occurred, and the patient was discharged from the hospital 2 weeks after surgery.

Histopathological examination showed characteristic findings of follicular occlusion. In the epidermis, the upper parts of the hair follicles were enlarged due to follicular keratosis and plugging; and in the dermis, acute inflammatory changes, as well as abscesses, were seen around the sinus tracts. Plasma cells and eosinophils were also present around the area, and a foreign body reaction due to a secondary inflammatory response was observed (Fig. 6).

Based on the clinical, paraclinical, and intraoperative findings, a diagnosis of DCS and FOT was made. Though synovitis-acne-pustulosis-hyperostosis-osteitis (SAPHO) syndrome was suspected because the patient had previously undergone bilateral hip replacement surgery for avascular necrosis of both hips, he did not show any unusual symptoms related to SAPHO syndrome. Following the surgery, the patient continued to take isotretinoin $40 \mathrm{mg}$ PO every 12 hours after con- 

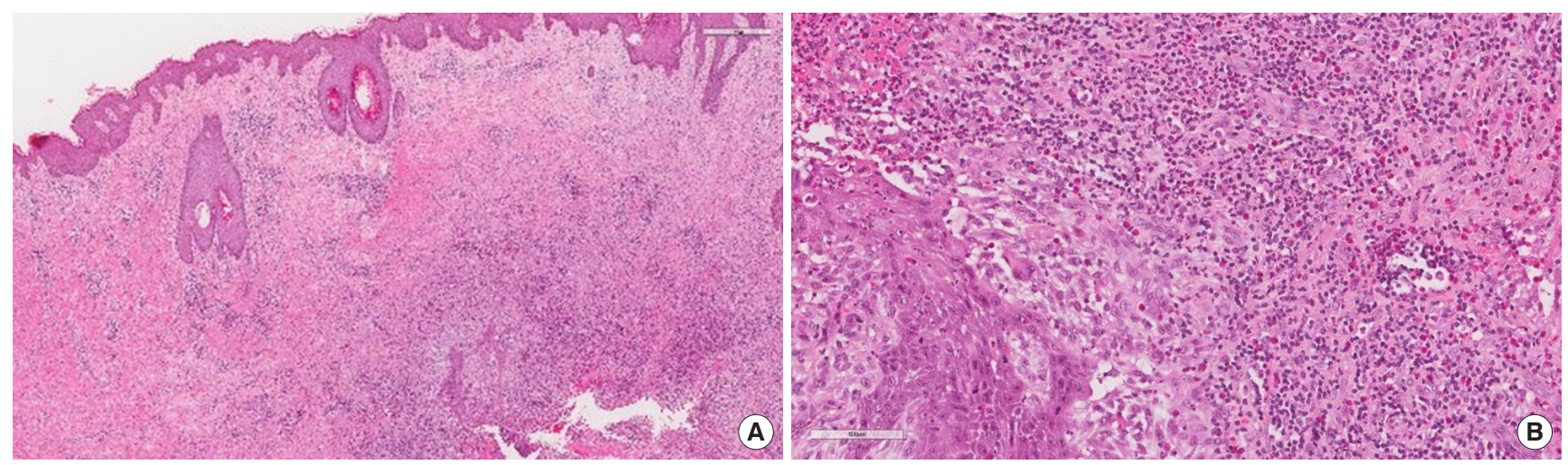

Fig. 6. Histopathological findings. (A) In the epidermis, upper parts of the hair follicles were enlarged due to follicular keratosis and plugging; in the dermis, acute inflammatory changes as well as abscesses were seen around the sinus tracts (H\&E, $\times 20)$. (B) Plasma cells and eosinophils were also present around the area, and foreign body reaction was observed as a result of secondary inflammatory reaction $(H \& E, \times 200)$.

sulting a dermatologist and has been on regular follow-up for 6 months without any complications or exacerbation of symptoms.

\section{Discussion}

The similarity in the pathophysiology of follicular occlusion involved in HS, AC, and DCS was first reported by Goeckermann in 1940 [2]. In 1952, Brunsting described the four common features of these conditions: glandular hyperplasia of the pilosebaceous apparatus or apocrine gland with hyperactivity, follicular occlusion and comedone formation, bacterial suppuration and undermined loose areolar tissue, and cicatricial healing [2]. In 1956, Pillsbury named the complex FOT for the first time [1].

The initial event common to all three conditions is follicular keratosis, which plugs the orifices of the pilosebaceous-apocrine unit [1]. The occluded follicles rupture and re-epithelialize, resulting in sinuses and fistulas [3]. These sinuses and fistulas become interconnected to each other and form the draining sinus tracts [3]. This results in the characteristic skin manifestations such as deep-seated nodules and comedones [1]. As these lesions expand, a honeycomb pattern develops, as seen in this case [4]. When the lesions undergo fibrosis and scarring, it might result in severe disfigurement and even cicatricial alopecia in cases of DCS [4-6]. It has also been reported that chronic lesions can develop into skin cancer due to malignant transformation $[4,5,7]$.

FOT is clinically diagnosed, and biopsy is not essential for the diagnosis [4]. The three components of FOT have similar skin manifestations [8]. However, the location of the lesions usually allows the differentiation of each $[4,8]$. When the skin lesions are located at an intertriginous area such as the axilla, groin, submammary fold, perineum, buttocks, or behind the ears, the condition can be diagnosed as HS; if the lesions are on the face, neck, chest, upper shoulders, arms, buttocks and thighs, the condition is most likely AC; and when the lesions are on the scalp, the condition is diagnosed as DCS $[4,8]$.

Regarding the pathogenesis, recently published research has focused on molecular or immunologic approaches, and the association between several factors has been reported. Factors including hypersensitivity to Cutibacterium acnes antigens, androgens, ingestion of halogens, chromosomal defects in the XYY syndrome, and other genetic factors such as NCSTN (nicastrin) have been suggested to be associated with AC [6]. Genetic factors, including NCSTN, PSEN1 (presenilin-1), and PSENEN (presenilin enhancer) genes related to $\mathrm{r}$-secretase production, immune dysregulation by proinflammatory cytokines like tumor necrosis factor (TNF)- $\alpha$, and interleukin (IL)$1 \beta$, and also IL-17 have been associated with the development of HS [3]. With proinflammatory cytokines being prominently associated with HS skin lesions and the same gene mutations appearing between HS and other autoinflammatory diseases, it is known that autoinflammation reaction plays a leading role in the pathogenesis of HS [9]. SAPHO syndrome, pyoderma gangrenosum-acne-suppurative hidradenitis (PASH) syndrome, pyoderma acne-pyoderma gangrenosum-acne-suppurative hidradenitis (PAPASH) syndrome, and pyoderma gangrenosum-acne vulgaris-suppurative hidradenitis-ankylosing spondylitis (PASS) syndrome have been reported as autoinflammatory syndromes related with HS and AC $[6,10]$. Since skin symptoms, such as HS lesion, are usually a leading sign of 
autoinflammatory disease in other organs, early detection based on the signs of systemic involvement and early treatment could delay the progression of the autoinflammatory process and prevent irreversible consequences [11].

Several chronic pyloritic hypersensitivity diseases, such as inflammatory bowel diseases, Crohn's disease, AdamantiadesBehçet disease, and spondylarthritis have been reported in patients with HS [11]. Arthritis, keratitis, pyoderma gangrenosum, and rarely, Crohn's disease, have been reported in patients with DCS [8]. Patients with FOT accompanied by pilonidal sinus may be diagnosed with follicular occlusion tetrad [3].

Treatment options for FOT should be tailored according to the status of lesions of each condition. Patient education about lifestyle modifications is essential for symptomatic relief in HS and $\mathrm{AC}$ since smoking cessation and weight reduction alone might improve the outcome of medical treatment, reduce the severity of the lesions, and prolong the remission period $[6,12]$. In case of superficial HS lesions, topical clindamycin has been reported to be effective for symptomatic relief [12]. In case of more severe or extensive lesions, clindamycin-rifampicin combination, tetracycline, minocycline, doxycycline, and acitretin are known to be effective [12]. Currently, in patients with moderate to severe HS who do not respond to conventional therapy, the use of adalimumab is approved [13]. Marzano et al. [13] reported that the more the adalimumab treatment is delayed for patients with HS, the weaker the response to the treatment. This supported the advantage of the early administration of adalimumab and the "window of opportunity" hypothesis in the treatment of HS patients.

Oral isotretinoin is known to be the first choice of therapy in patients with AC; additional use of systemic glucocorticoids or dapsone have also been reported to be helpful [6]. In general, monotherapy agents for common acne such as tetracycline, doxycycline, minocycline, or azithromycin do not achieve good treatment outcomes in patients with AC. When there is no response to isotretinoin, other options that have been reported to be effective include colchicine, cyclosporine, gonadotropin-releasing hormone analogues such as buserelin, and TNF- $\alpha$ antagonists [6].

Oral antibiotics such as doxycycline, azithromycin, rifampicin form the first line treatment for mild DCS [14]. For severe DCS that does not respond to antibiotic therapy, isotretinoin is usually used for treatment. Guo et al. [14] conducted a meta-analysis of the adverse effects of isotretinoin treatment and reported that isotretinoin relieves the symptoms of DCS. Laser or intense pulsed light hair removal, Nd:YAG pulsed diode, or
$\mathrm{CO}_{2}$ laser, and photodynamic therapy have been used for the treatment of localized lesions of FOT $[5,6,12]$.

For the patients who do not respond to medication or develop repeated recurrence, surgical treatment is considered.

Biofilm formation is seen in chronic HS lesions, interfering with antibiotic treatment and causing chronic disease [15]. For the removal of otherwise intractable biofilms, debridement or excision of the chronic lesion, incision and drainage, or even simple deroofing can be an effective procedure [15]. For curative treatment, the skin and soft tissue contained in the lesion must be radically excised $[5,6,12]$. Although radical wide excision can minimize recurrences at the same site, it is difficult to perform in case of an extensive disease involving multiple sites, and a longer duration of treatment is necessary for the reconstruction of large-area defects following wide excision. Further disfigurement might occur because of the surgical treatment, while the surgical measures cannot prevent new disease progression in other areas [12].

In the present case, the lesions of $\mathrm{HS}$ and $\mathrm{AC}$ remained unchanged, with no exacerbations following medical treatment, and the DCS lesion remained localized to the occipital area. Since the patient was relieved of his acute stage symptoms following incision and drainage, and the earlier lesions remained unchanged, aggressive treatment was not necessary as it could prolong the duration of treatment and affect the patient's quality of life. Thus, the patient was maintained on isotretinoin 40 mg PO every 12 hours after consultation with a dermatologist and has been on regular follow-up at the outpatient clinic for signs of drug adverse effects, exacerbations, and malignant changes in the lesions.

We have presented a case report with literature review in the hope that the presented FOT treatments will become a potential treatment option in the future.

\section{Conflict of interest}

This study was supported by the Research Funds of Dong-A University. Otherwise, no potential conflict of interest relevant to this article was reported.

\section{ORCID iDs}

Chung-Min Yoon https://orcid.org/0000-0003-0307-5545

Jung-Ha Kwak https://orcid.org/0000-0003-3079-9223

Song-Hee Han https://orcid.org/0000-0002-4564-7014

Ji-An Choi https://orcid.org/0000-0003-4125-8043 


\section{References}

1. Montes LF, Curtis AC. The follicular occlusion triad. Postgrad Med 1968;43:108-12.

2. Chicarilli ZN. Follicular occlusion triad: hidradenitis suppurativa, acne conglobata, and dissecting cellulitis of the scalp. Ann Plast Surg 1987;18:230-7.

3. Goldburg SR, Strober BE, Payette MJ. Hidradenitis suppurativa: epidemiology, clinical presentation, and pathogenesis. J Am Acad Dermatol 2020;82:1045-58.

4. Alikhan A, Lynch PJ, Eisen DB. Hidradenitis suppurativa: a comprehensive review. J Am Acad Dermatol 2009;60:53961.

5. Thomas J, Aguh C. Approach to treatment of refractory dissecting cellulitis of the scalp: a systematic review. J Dermatolog Treat 2021;32:144-9.

6. Canpolat F, Kurmus GI, Gonul M. Acne conglobata. Rom J Clin Exp Dermatol 2017;2:68-73.

7. Curry SS, Gaither DH, King LE Jr. Squamous cell carcinoma arising in dissecting perifolliculitis of the scalp: a case report and review of secondary squamous cell carcinomas. J Am Acad Dermatol 1981;4:673-8.

8. Syed TA, Ul Abideen Asad Z, Salem G, et al. Dissecting cellulitis of the scalp: a rare dermatological manifestation of Crohn's disease. ACG Case Rep J 2018;5:e8.

9. Zouboulis CC, Benhadou F, Byrd AS, et al. What causes hidradenitis suppurativa?-15 years after. Exp Dermatol 2020; 29:1154-70.

10. Nikolakis G, Kaleta KP, Vaiopoulos AG, et al. Phenotypes and pathophysiology of syndromic hidradenitis suppurativa: different faces of the same disease? A systematic review. Dermatology 2020 Sep 17 [Epub]. https://doi.org/10.1159/000509873.

11. Fimmel S, Zouboulis CC. Comorbidities of hidradenitis suppurativa (acne inversa). Dermatoendocrinol 2010;2:9-16.

12. Scuderi N, Monfrecola A, Dessy LA, et al. Medical and surgical treatment of hidradenitis suppurativa: a review. Skin Appendage Disord 2017;3:95-110.

13. Marzano AV, Genovese G, Casazza G, et al. Evidence for a 'window of opportunity' in hidradenitis suppurativa treated with adalimumab: a retrospective, real-life multicentre cohort study. Br J Dermatol 2021;184:133-40.

14. Guo W, Zhu C, Stevens G, et al. Analyzing the efficacy of isotretinoin in treating dissecting cellulitis: a literature review and meta-analysis. Drugs R D 2021;21:29-37.

15. Ring HC, Bay L, Nilsson M, et al. Bacterial biofilm in chronic lesions of hidradenitis suppurativa. Br J Dermatol 2017;176:993-1000. 